\title{
Nota
}

\section{O USO DE AMOSTRAS PENEIRADAS A DOIS MILÍMETROS SUBESTIMA A PROTEÇÃO FÍSICA DA MATÉRIA ORGÂNICA NO SOLO(1)}

\author{
Michely Tomazi $^{(2)}$, Cimélio Bayer ${ }^{(3)} \&$ Paulo César Conceição ${ }^{(4)}$
}

\begin{abstract}
RESUMO
O fracionamento físico densimétrico é uma importante ferramenta no estudo da proteção física da matéria orgânica (MO) em agregados de solo, porém seus resultados podem ser alterados pela malha da peneira utilizada no preparo da amostra. A hipótese do presente estudo é de que a desagregação excessiva do solo pelo uso de peneira de $2 \mathrm{~mm}$ libera MO originalmente protegida dentro de agregados e, portanto, subestima o teor de C na fração leve-oclusa (C-FLO) e a magnitude da proteção física da MO intra-agregados, em comparação à peneira de $\mathbf{9 , 5} \mathbf{m m}$. Amostras (0-5 cm) de cinco solos foram coletadas em experimentos de longa duração (9-18 anos) nas regiões Centro-Oeste (quatro solos) e Sul do Brasil (um solo), sob sistemas de manejo convencional e conservacionista. Tomando as amostras $\leq 9,5 \mathrm{~mm}$ como referência, verificou-se subestimação de 2,9 a $15,7 \%$ no C-FLO quando utilizadas amostras $\leq 2,0 \mathrm{~mm}$, a qual foi mais expressiva nos sistemas conservacionistas de manejo de solo e positivamente correlacionada com os teores de $\mathrm{C}$ no solo $(\mathrm{r}=0,92, \mathrm{p}<0,04)$, massa de macroagregados $>2 \mathrm{~mm}(\mathrm{r}=0,90, \mathrm{p}<0,02)$ e índice de estabilidade de agregados $(r=0,94, p<0,01)$. Em três dos cinco solos estudados, o uso de amostras $\leq 2,0 \mathrm{~mm}$ levou à conclusão errônea de que os sistemas conservacionistas de manejo do solo não são efetivos na proteção física da MO do solo. Em estudos da proteção física da MO intra-agregados indica-se o uso da peneira de malha de 9,5 $\mathrm{mm}$ no preparo da amostra ou, alternativamente, da mesma malha da peneira empregada na preparação de amostras para avaliação da estabilidade de agregados, usualmente $8 \mathrm{~mm}$ ou maior.
\end{abstract}

Termos de indexação: fracionamento físico, sistema de manejo conservacionista, fração leve-oclusa.

\footnotetext{
(1) Recebido para publicação em 20 de janeiro de 2010 e aprovado em 4 de abril de 2011.

(2) Bolsista Prodoc do Programa de Pós-Graduação em Ciência do Solo da Universidade Federal do Rio Grande do Sul - UFRGS. Av. Bento Gonçalves 7712, CEP 91540-000 Porto Alegre (RS). E-mail: mitomazi@yahoo.com.br

(3) Professor do Departamento de Solos, UFRGS. Pesquisador do CNPq. E.mail: cimelio.bayer@ufrgs.br

(4) Professor da Universidade Tecnológica do Paraná, Campus Dois Vizinhos - UTFPR-DV. Estrada para Boa Esperança, CEP 85660 000 Dois Vizinhos (PR). Pesquisador do CNPq. E-mail: pcconceicao@hotmail.com
} 


\title{
SUMMARY: PHYSICAL PROTECTION OF SOIL ORGANIC MATTER IS UNDERESTIMATED IN TWO MILLIMETER- SIEVED SOIL
}

\begin{abstract}
Density fractionation is an important tool to determine the physical protection of organic matter within soil aggregates, but results may be affected by the mesh size used to sieve soil samples. The hypothesis of this study is that in comparison to $9.5 \mathrm{~mm}$ sieve, sieving through $2 \mathrm{~mm}$ mesh results in excessive soil disaggregation by releasing organic matter originally protected within aggregates and consequently leads to underestimations of the carbon content in the occluded light fraction $(O L F-C)$ and of the magnitude of physical protection of organic matter within soil aggregates. Soil samples $(0-5 \mathrm{~cm})$ of five Brazilian soils under long-term experiments (9-18 yrs) were collected in the regions Center West (4 soils) and South (1 soil), under conventional and conservation soil management systems. Based on $\leq 9.5 \mathrm{~mm}$ soil samples as reference, an underestimation of 2.9-15.7\% of the OLF-C pool was observed in comparison to $\leq 2.0$ soil samples $\mathrm{mm}$. This effect was more pronounced for conservation soil management systems and positively correlated with soil organic $C$ content $(r=0.92, p<0.04)$, macroaggregate mass $>2 \mathrm{~mm}(r=0.90, p<0.02)$ and aggregate stability index $(r=0.94$, $p<0.01$ ). In three of the evaluated soils, the use of $\leq 2.0 \mathrm{~mm}$ soil samples led to the erroneous conclusion that conservation soil management systems are not efficient to stabilize organic matter within soil aggregates. Therefore, a mesh size of $9.5 \mathrm{~mm}$ is indicated to evaluate the physical protection of organic matter in aggregates, or alternatively, the sieves used to prepare soil samples for aggregate stability analysis, i.e., usually greater than $8 \mathrm{~mm}$.
\end{abstract}

Index terms: physical fractionation, conservation management system, light occluded.

\section{INTRODUÇÃO}

Os métodos de fracionamento físico têm sido amplamente utilizados no estudo da dinâmica e qualidade da matéria orgânica (MO) sob diferentes tipos de uso e manejo do solo (Freixo et al., 2002; Roscoe \& Burman, 2003; Dieckow et al., 2005; Bayer et al., 2004, 2006; Conceição et al., 2008). Especificamente, o fracionamento densimétrico baseia-se na separação de frações orgânicas com base na densidade das partículas, obtendo-se a fração leve (FL), que corresponde à MO não associada aos minerais, e a fração pesada (FP), a qual é associada aos minerais e, por isso, apresenta maior densidade (Christensen, 1992; Golchin et al., 1994a; Roscoe \& Machado, 2002; Conceição et al, 2008). A FL pode ser separada em FL-livre ou interagregados (FLL) e FL-oclusa ou intra-agregados (FLO). Estas três frações (FLL, FLO e FP) apresentam diferentes funções e permitem fazer inferências sobre a atuação dos mecanismos de estabilização da MO no solo (estabilidade química, proteção física e estabilidade coloidal) (Christensen, 1992; Golchin et al., 1994a,b; Lützow et al., 2007; Conceição et al., 2008).

Avanços metodológicos têm contribuído para melhorar a eficiência do fracionamento físico densimétrico, como a adequação de produtos e da densidade das soluções, formas de dispersão do solo, entre outros. Os produtos mais utilizados são o iodeto de sódio (NaI) e o politungstato de sódio (PTS), sendo o último mais eficiente na recuperação da FLO (Conceição et al., 2007). Entretanto, pouca atenção tem sido dada ao preparo da amostra, especificamente ao tamanho da malha da peneira usada para delimitar o tamanho máximo dos agregados que compõem a amostra a ser utilizada no fracionamento.
Em trabalhos disponíveis na literatura, por exemplo, verifica-se o preparo de amostras em peneiras com malha variando de 2 a $19 \mathrm{~mm}$ (Golchin et al., 1994a; Roscoe \& Machado, 2002; Roscoe et al., 2006; Conceição et al., 2007, 2008). Entretanto, solos tropicais e subtropicais, como os Argissolos e Latossolos argilosos, com medianos a elevados teores de óxidos de ferro, apresentam em geral mais que $50 \%$ da massa de agregados com diâmetro maior que $2 \mathrm{~mm}$, principalmente quando sob vegetação natural ou sistemas de manejo conservacionista (Silva \& Mielniczuk, 1997; Conceição et al., 2005; Madari et al., 2005; Salton et al., 2008). Nesses solos, o preparo da amostra em peneira com malha de $2 \mathrm{~mm}$ pode subestimar o teor de C na FLO, pois a desagregação prévia do solo possivelmente irá liberar parte da MO intra-agregados $>2 \mathrm{~mm}$, a qual seria quantificada com FLL.

Possivelmente, esse efeito seja dependente das características dos solos, sobretudo daquelas que influenciam a estabilidade de agregados. Devido à alta relação da FLO com a agregação do solo (Conceição, 2006), espera-se que, quanto maior a quantidade de agregados grandes, maior seja a subestimação da FLO. No entanto, como existem vários fatores envolvidos na agregação, os solos podem responder diferentemente ao efeito do preparo da amostra.

O presente estudo teve como objetivo avaliar o impacto do preparo inicial da amostra, no que se refere à malha da peneira utilizada $(2,0$ e $9,5 \mathrm{~mm})$, sobre a quantificação das frações FLL e FLO obtidas no fracionamento densimétrico da $\mathrm{MO}$ em cinco solos brasileiros sob sistemas de manejo convencional e conservacionista. 


\section{MATERIAL E MÉTODOS}

O estudo foi realizado em amostras da camada superficial dos solos $(0-5 \mathrm{~cm})$, a qual é a mais afetada pelos sistemas de manejo no que se refere aos compartimentos da MO (Conceição et al., 2008). Em cada solo foram selecionados dois sistemas de manejo com alto e baixo potencial de acúmulo de $\mathrm{C}$ e agregação em experimentos de longa duração (9-18 anos), sendo quatro localizados na região Centro-Oeste e um na região Sul do Brasil (Quadro 1). Esses solos apresentam ampla variação do teor de argila (220$861 \mathrm{~g} \mathrm{~kg}^{-1}$ solo), mineralogia (cauliníticos e oxídicos), teor de C orgânico $\left(9,1-40,3 \mathrm{~g} \mathrm{~kg}^{-1}\right)$, massa de agregados $>2 \mathrm{~mm}(12-72 \%)$, diâmetro médio ponderado (DMP, 2,04-4,86 $\mathrm{mm}$ ) e índice de estabilidade de agregados (IEA, 60-100 \%) (Quadros 1 e 2).

Em cada solo e sistema de manejo, foram coletados blocos indeformados (20 × 20 × $5 \mathrm{~cm})$ em triplicata, com auxílio de pá de corte e espátula. As amostras foram transportadas ao laboratório, desagregadas manualmente até toda a massa de solo passar pela peneira de 9,5 mm e secas à sombra por $72 \mathrm{~h}$.

Para cada tratamento, separaram-se duas subamostras de $10 \mathrm{~g}$. Uma delas, passada em peneira de $9,5 \mathrm{~mm}$, foi utilizada diretamente para o fracionamento físico da MO, enquanto a outra foi destorroada com rolo de madeira até passar em peneira de malha de $2 \mathrm{~mm}$. Nesse processo, todos os resíduos orgânicos liberados pela quebra dos agregados foram forçados a passar na peneira, visando manter a mesma quantidade de $\mathrm{MO}$ da amostra original.

Ambas as subamostras de $10 \mathrm{~g}$, com agregados $\leq 9,5 \mathrm{e} \leq 2,0 \mathrm{~mm}$, foram submetidas ao fracionamento físico densimétrico da MO para obtenção das frações FLL e FLO. Os $10 \mathrm{~g}$ de solo foram colocados em tubo de centrífuga $(100 \mathrm{~mL})$ contendo $80 \mathrm{~mL}$ de PTS $\left(2 \mathrm{~kg} \mathrm{~L}^{-1}\right)$, o qual foi vedado com rolha de borracha e invertido lenta e manualmente por cinco vezes, para liberação da FLL da massa de solo, com um mínimo rompimento dos agregados. A suspensão foi centrifugada a $2.000 \mathrm{~g}$ por $90 \mathrm{~min}$ e, posteriormente, o sobrenadante contendo a FLL foi vertido em sistema de filtração sob vácuo com filtro de fibra de vidro (Whatman GF/A, 0,45 $\mu \mathrm{m}$ ) previamente seco em estufa $\left(50^{\circ} \mathrm{C}\right)$ e pesado (Conceição et al., 2007).

A solução de PTS que passou pelo filtro retornou ao tubo de centrífuga contendo o pellet (FLO+FP) e a suspensão foi submetida a ultrassom, para dispersão dos agregados do solo e, consequentemente, liberação da FLO (MOS intra-agregados e não associadas aos minerais). A energia de ultrassom utilizada na dispersão dos solos variou de 250 a $800 \mathrm{~kJ} \mathrm{~L}^{-1}$ (Quadro 1) e consistiu na energia necessária para obtenção de 99 \% da fração argila do solo, determinada a partir de uma curva de dispersão de argila obtida com a aplicação de energias crescentes (Inda Junior et al., 2007). A recuperação da FLO foi realizada por centrifugação e filtragem, assim como descrito para a FLL.

\section{Quadro1. Dados gerais dos solos utilizados nos testes de fracionamento físico}

\begin{tabular}{|c|c|c|c|c|c|c|c|c|c|}
\hline \multirow{2}{*}{$\begin{array}{l}\text { Solo/ } \\
\text { local }^{(1)}\end{array}$} & \multirow{2}{*}{$\begin{array}{l}\text { Duração do } \\
\text { experimento }\end{array}$} & \multirow{2}{*}{$\begin{array}{l}\text { Uso do solo anterior } \\
\text { ao experimento }\end{array}$} & \multirow{2}{*}{$\begin{array}{c}\text { Temperatura } \\
\text { média } \\
\text { anual }\end{array}$} & \multirow{2}{*}{$\begin{array}{c}\text { Precipitação } \\
\text { média } \\
\text { anual }\end{array}$} & \multirow{2}{*}{ Argila } & \multirow{2}{*}{$\begin{array}{c}\text { Energia } \\
\text { dispersão }^{(2)}\end{array}$} & \multirow{2}{*}{$\begin{array}{c}\text { Minerais } \\
\text { predominantes }^{(3)}\end{array}$} & \multicolumn{2}{|c|}{ Sistemas de manejo do solo ${ }^{(4)}$} \\
\hline & & & & & & & & Alto PAC & Baixo PAC \\
\hline & anos & & ${ }^{\circ} \mathrm{C}$ & $\mathrm{mm}$ & $\mathrm{g} \mathrm{kg}^{-1}$ & $\mathrm{~kJ} \mathrm{~L} \mathrm{~L}^{-1}$ & & & \\
\hline PV - ES & 18 & 15 anos de $\mathrm{PC}$ & 19,4 & 1440 & 220 & 250 & $\mathrm{Ct}, \mathrm{Hm}$ & $\mathrm{PC}-\mathrm{A} / \mathrm{M}$ & $\mathrm{PD}-\mathrm{A}+\mathrm{V} / \mathrm{M}+\mathrm{C}$ \\
\hline $\mathrm{LV} \cdot \mathrm{CG}$ & 11 & $\begin{array}{l}20 \text { anos de } \\
\text { PP - } \mathrm{B} d \text { degradada }\end{array}$ & 23 & 1635 & 360 & 800 & $\mathrm{Ct}, \mathrm{Gb}, \mathrm{Hm}$ & PC - S & $\mathrm{PP}(\mathrm{B} d)$ \\
\hline $\mathrm{LV} \cdot \mathrm{D}$ & 9 & $\begin{array}{l}20 \text { anos de PC } \\
\text { (arroz, milho, soja) }\end{array}$ & 23 & 1545 & 670 & 450 & $\mathrm{Ct}, \mathrm{Hm}$ & $\mathrm{PD} \cdot \mathrm{NM} / \mathrm{AS} / \mathrm{TS}$ & $\mathrm{PP}(\mathrm{B} d)$ \\
\hline $\mathrm{LV} \cdot \mathrm{M}$ & 11 & 1 ano de $\mathrm{PC}$ & 23 & 1527 & 530 & 530 & $\mathrm{Ct}, \mathrm{Hm}$ & $\mathrm{PD}-\mathrm{S} / \mathrm{A}$ & $\mathrm{PP}(\mathrm{B} d+\mathrm{Sg})$ \\
\hline LV - CR & 13 & 19 anos em PC & 24 & 2349 & 861 & 407 & $\mathrm{Ct}, \mathrm{Gb}, \mathrm{Hm}$ & $\mathrm{PC}-\mathrm{S} / \mathrm{A}$ & PD - S/A \\
\hline
\end{tabular}

(1) PV-ES: Argissolo Vermelho (Eldorado do Sul - RS); LV - Latossolo Vermelho em Campo Grande - MS (CG), Dourados - MS (D), Maracaju, MS (M) e Costa Rica - MS (CR). ${ }^{(2)}$ Energia de dispersão $\left(\mathrm{J} \mathrm{mL}^{-1}\right)$ aplicada para obtenção de $99 \%$ da fração argila em cada solo. ${ }^{(3)} \mathrm{Ct}$ : caulinita, Hm: hematita, Gb: gibsita. ${ }^{(4)}$ Alto PAC: alto potencial de acúmulo de C; Baixo PAC: baixo potencial de acúmulo de C; PC-A/M: preparo convencional associado ao sistema de cultura aveia-preta (Avena strigosa) no inverno e milho (Zea mays) no verão; PD-A+V/M+C: plantio direto associado ao sistema aveia + vica (Vicia sativa L.) no inverno e milho + caupi (Vigna unguiculata (L) Walp) no verão; PC-S: preparo convencional com pousio no inverno e soja (Glycine max) no verão; PP $(\mathrm{B} d)$ : pastagem permanente de gramínea (Brachiaria decumbens); PD-NM/AS/TS: plantio direto associado ao sistema de rotação de culturas trianual incluindo N=nabo forrageiro (Raphanus sativus), M: milho, A: aveia-preta, S: soja (Glycine max) e T: trigo (Triticum aestivum); PD-S/A: plantio direto associado ao sistema de cultura aveia-preta no inverno e soja no verão; $\mathrm{PP}(\mathrm{Bd}+\mathrm{Sg})$ : pastagem permanente de gramínea (Brachiaria decumbens) consorciada com leguminosa (Stylosanthes guianensis); PC-S/A: preparo convencional associado ao sistema de cultura soja/algodão; PD-S/A: plantio direto associado ao sistema de cultura soja/ algodão. Dados compilados de diferentes fontes: Boeni (2007); Inda Junior et al. (2007); Conceição et al. (2008); Salton et al. (2008); Tomazi (2008). 
Quadro 2. Teores de C orgânico total (COT), diâmetro médio ponderado (DMP), agregados $>2$ mm e índice de estabilidade de agregados (IEA) nos diferentes solos sob sistemas de manejo avaliados com alto e baixo potencial de acúmulo de C (PAC)

\begin{tabular}{|c|c|c|c|c|c|}
\hline Tipo de solo & PAC & C orgânico & DMP & Agregados $>2 \mathrm{~mm}$ & IEA \\
\hline & & $\mathrm{g} \mathrm{kg}^{-1}$ solo & $\mathrm{mm}$ & $\longrightarrow \%$ & - \\
\hline PV-ES & $\begin{array}{l}\text { Baixo } \\
\text { Alto }\end{array}$ & $\begin{array}{r}9,1 \\
18,9\end{array}$ & $\begin{array}{l}2,04 \\
2,67\end{array}$ & $\begin{array}{l}29 \\
59\end{array}$ & $\begin{array}{l}63 \\
95\end{array}$ \\
\hline $\mathrm{LV}-\mathrm{CG}$ & $\begin{array}{l}\text { Baixo } \\
\text { Alto }\end{array}$ & $\begin{array}{l}16,5 \\
27,8\end{array}$ & $\begin{array}{l}0,95 \\
3,30\end{array}$ & $\begin{array}{l}12 \\
55\end{array}$ & $\begin{array}{r}60 \\
100\end{array}$ \\
\hline LV-D & $\begin{array}{l}\text { Baixo } \\
\text { Alto }\end{array}$ & $\begin{array}{l}18,2 \\
30,5\end{array}$ & $\begin{array}{l}2,56 \\
3,95\end{array}$ & $\begin{array}{l}39 \\
62\end{array}$ & $\begin{array}{l}71 \\
91\end{array}$ \\
\hline LV-M & $\begin{array}{l}\text { Baixo } \\
\text { Alto }\end{array}$ & $\begin{array}{l}22,4 \\
35,7\end{array}$ & $\begin{array}{l}2,21 \\
4,86\end{array}$ & $\begin{array}{l}34 \\
72\end{array}$ & $\begin{array}{r}76 \\
100\end{array}$ \\
\hline LV - CR & $\begin{array}{l}\text { Baixo } \\
\text { Alto }\end{array}$ & $\begin{array}{l}27,3 \\
40,3\end{array}$ & $\begin{array}{l}2,04 \\
2,67\end{array}$ & $\begin{array}{l}30 \\
40\end{array}$ & $\begin{array}{l}63 \\
74\end{array}$ \\
\hline
\end{tabular}

PV-ES: Argissolo Vermelho (Eldorado do Sul - RS); LV: Latossolo Vermelho em Campo Grande - MS (CG), Dourados - MS (D), Maracaju - MS (M) e Costa Rica - MS (CR). Fonte: Boeni (2007); Conceição (2006); Salton et al. (2008); Tomazi (2008).

Os filtros contendo a fração FLL ou FLO foram lavados sequencialmente com $200 \mathrm{~mL}$ de água destilada e $50 \mathrm{~mL}$ de solução de $\mathrm{CaCl}_{2}\left(10 \mathrm{mmol} \mathrm{L}^{-1}\right)$ para remover o PTS, e $200 \mathrm{~mL}$ de água destilada, para remover o $\mathrm{CaCl}_{2}$ das frações. Cada filtro, juntamente com a FLL ou FLO, foi seco em estufa a $50{ }^{\circ} \mathrm{C}$ por $24 \mathrm{~h}$, pesado e moído em grau de ágata. Devido à ausência de $\mathrm{C}$ inorgânico nesses solos altamente intemperizados e ácidos, o teor de C orgânico do solo e das diferentes frações obtidas (FLL e FLO) foi determinado por combustão seca a $900{ }^{\circ} \mathrm{C}$, em um analisador Shimadzu (modelo VCSH).

Os resultados foram submetidos à análise da variância, sendo a comparação das médias do teor de $\mathrm{C}$ nas frações FLL e FLO, obtidas das amostras $\leq 2,0$ $\mathrm{e} \leq 9,5 \mathrm{~mm}$, feita pelo teste te Student $(\mathrm{p}<0,05)$. A correlação de Pearson foi utilizada para verificar a relação entre a subestimação do C-FLO com uso de amostras $\leq 2,0 \mathrm{~mm}$ e as variáveis de solo (teor de $\mathrm{C}$ orgânico, massa de agregados $>2 \mathrm{~mm}$, DMP e IEA).

\section{RESULTADOS E DISCUSSÃO}

\section{Teores de C das frações leve-livre (FLL) e leve- oclusa (FLO)}

O teor de C da FLL e FLO foi influenciado pela malha da peneira utilizada (2,0 ou $9,5 \mathrm{~mm})$. Em três dos cinco solos avaliados, a concentração de C da FLL foi maior na amostra $\leq 2,0 \mathrm{~mm}$ (Quadro 3), principalmente para os sistemas de manejo com alto potencial de acúmulo de C. Isso indica que parte da FLO, a qual apresenta maior teor de C (Conceição et al., 2008; Golchin et al., 2004a), foi recuperada como FLL, devido à quebra dos agregados $>2 \mathrm{~mm}$.
Além disso, no LV-CG a FLO também apresentou maior teor de $\mathrm{C}$ na amostra $\leq 2,0 \mathrm{~mm}$. Esse possível enriquecimento em $\mathrm{C}$ reforça a hipótese discutida para FLL, visto que a quebra dos agregados estáveis $>2 \mathrm{~mm}$ durante o preparo da amostra liberaria a parte da FLO menos rica em C, o que é possível quando se considera a formação de agregados maiores a partir de agregados menores (Oades \& Waters, 1991). A FLO que possivelmente foi liberada dos agregados de tamanho entre 9,5 e 2,0 $\mathrm{mm}$, durante a moagem, estaria em um estádio inicial de decomposição e, por isso, menos rica em $\mathrm{C}$ do que a FLO dos agregados $\leq 2,0 \mathrm{~mm}$ (Skjemstad et al., 1996). Dessa forma, após a "migração" da FLO, menos rica em C, para a FLL, houve aumento no teor de $\mathrm{C}$ da FLO restante.

\section{Carbono do solo na forma de fração leve-livre (C-FLL) e leve-oclusa (C-FLO)}

De maneira geral, a utilização de amostras $\leq 2,0 \mathrm{~mm}$ subestimou a quantidade de C-FLO e, consequentemente, aumentou a quantidade de C-FLL, em comparação ao uso de amostras $\leq 9,5 \mathrm{~mm}$ (Quadro 4). Além disso, no solo LV-CG o sistema com baixo aporte de $\mathrm{C}$ apresentou maior quantidade de $\mathrm{C}$ FLL do que de C-FLO, em amostras $\leq 2,0 \mathrm{~mm}$. Esse comportamento não é coerente com a teoria de estabilização da MO, que prevê maior acúmulo de C na FLO (Conceição et al., 2008), decorrente da sua proteção física no interior dos agregados - um mecanismo adicional de estabilização da MO comparado ao C-FLL (Golchin et al., 1994b).

Em valores absolutos, a subestimação com o uso de amostras $\leq 2,0 \mathrm{~mm}$ variou de 0,04 a $1,43 \mathrm{~g} \mathrm{~kg}^{-1}$ $(\bar{x}=0,71)$ (Quadro 4), representando de 2,9 a $15,7 \%$ do total de $\mathrm{C}$ obtido na forma de fração leve total (CFLL + C-FLO). Essa subestimação mostrou correlação 
Quadro 3. Teores de C da fração leve-livre (FLL) e da fração leve-oclusa (FLO) obtidas no fracionamento físico densimétrico aplicado em amostras de solo $\leq 9,5 \mathrm{e} \leq 2,0 \mathrm{~mm}$, provenientes de cinco solos sob sistemas de manejo com alto e baixo potencial de acúmulo de C (PAC)

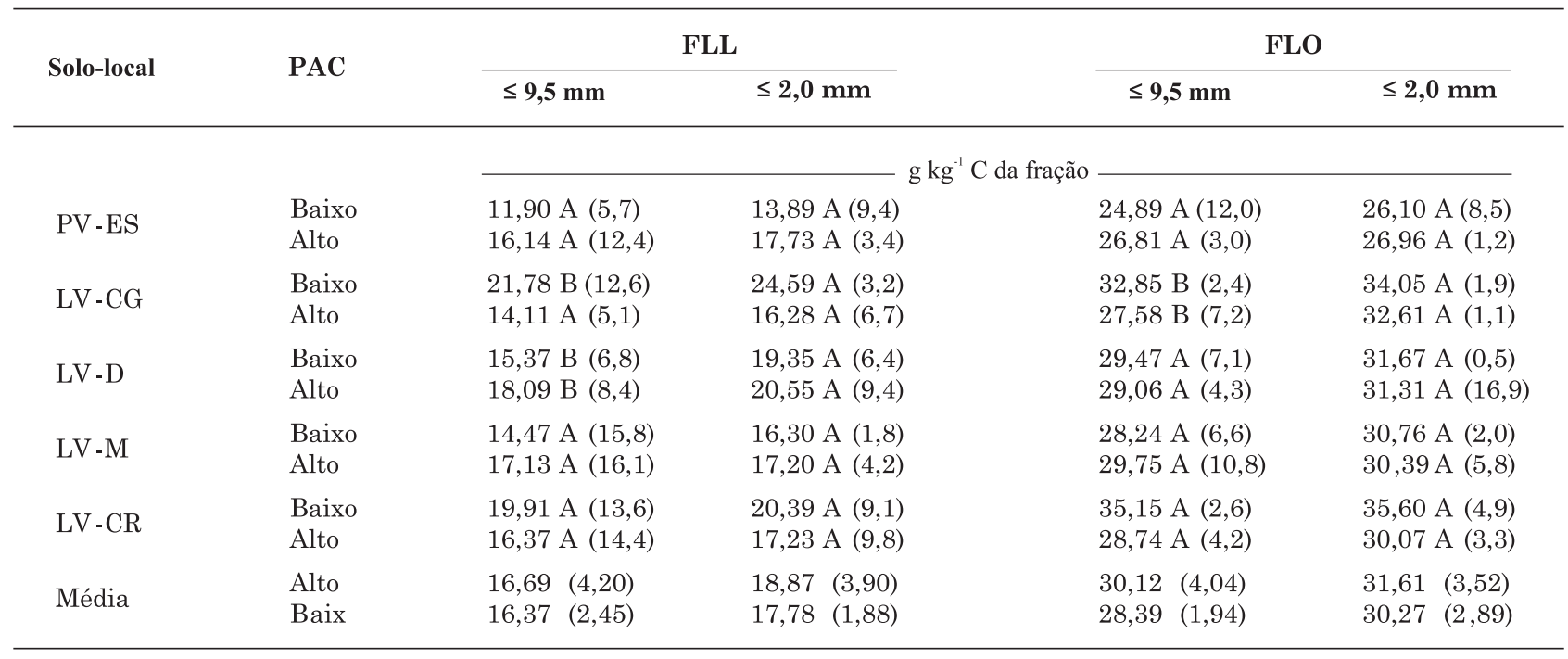

PV-ES: Argissolo Vermelho (Eldorado do Sul - RS); LV: Latossolo Vermelho em Campo Grande - MS (CG), Dourados - MS (D), Maracaju - MS (M) e Costa Rica - MS (CR). Valores seguidos de mesma letra, na linha, não diferem estatisticamente pelo teste $t$ de Student $(p<0,05)$. Valores entre parênteses são o coeficiente de variação expresso em percentagem $(n=3)$.

Quadro 4. Carbono do solo na forma de fração leve-livre (C-FLL), fração leve-oclusa (C-FLO), fração leve total (FL=FLL+FLO), e a variação de C-FLO obtida pelo fracionamento físico densimétrico aplicado a amostras $\leq 9,5$ e $\leq 2,0 \mathrm{~mm}$ de cinco solos sob sistemas de manejo com alto e baixo potencial de acúmulo de C (PAC)

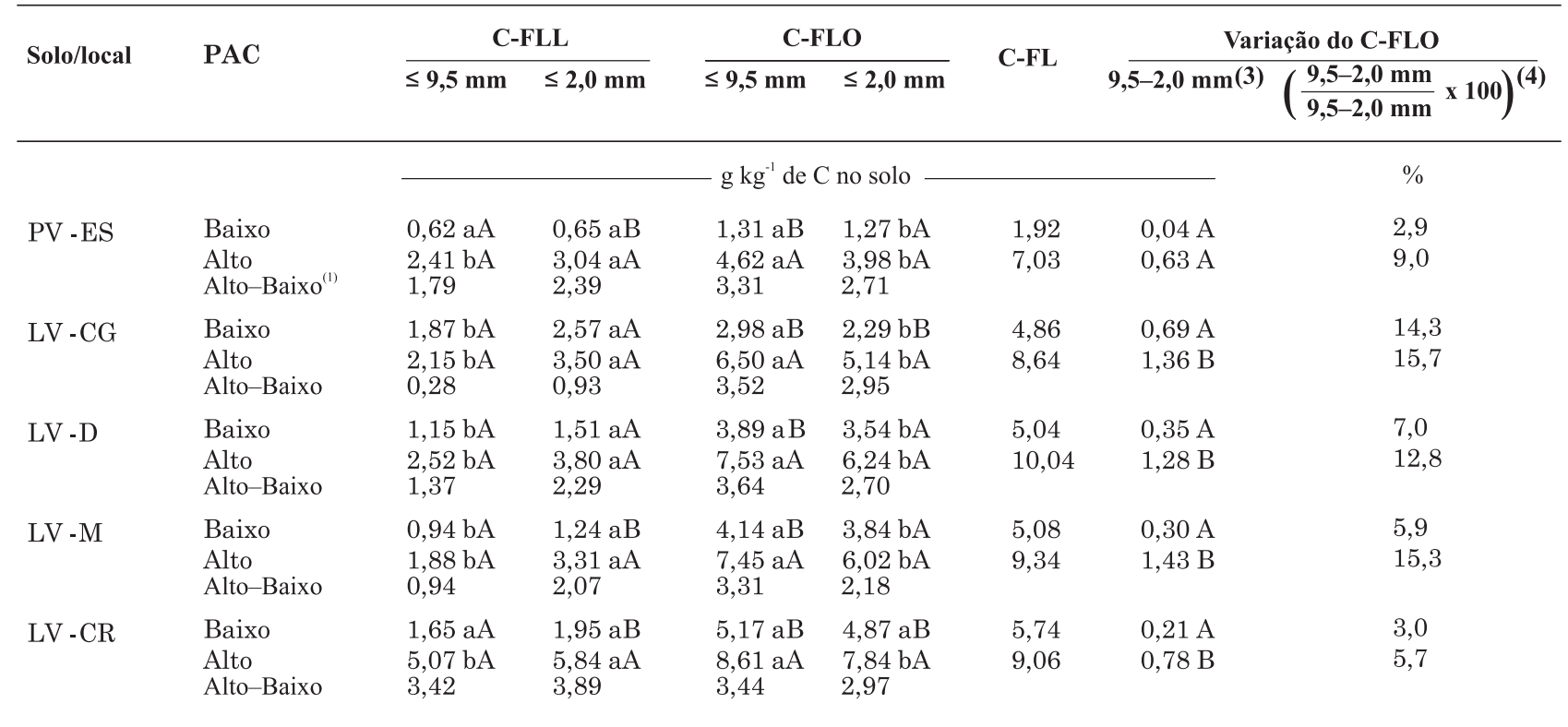

(1) Alto-baixo: diferença entre sistema com alto e com baixo potencial de acúmulo de $\mathrm{C}$ no solo. ${ }^{(2)} 9,5-2,0 \mathrm{~mm}=$ diferença entre CFLO em amostras $\leq 9,5$ e $\leq 2,0 \mathrm{~mm}$. (3) 9,5-2,0 mm: diferença entre C-FLO de amostras $\leq 9,5$ e $\leq 2,0 \mathrm{~mm}$. (4) $9,5-2,0 \mathrm{~mm} / \mathrm{C}-\mathrm{FL}$ : relação entre a diferença de C-FLO de amostras $\leq 9,5$ e $\leq 2,0 \mathrm{~mm}$ e o total de C na fração leve (C-FL) multiplicado por 100 . PV ES: Argissolo Vermelho (Eldorado do Sul - RS); LV: Latossolo Vermelho em Campo Grande - MS (CG), Dourados - MS (D), Maracajú, MS (M) e Costa Rica - MS (CR). Valores seguidos de mesma letra minúscula, na linha, e maiúscula, na coluna, não diferem estatisticamente pelo teste de $\mathrm{t}$ de Student $(\mathrm{p}<0,05)$.

significativa e positiva com o teor de $\mathrm{C}$ orgânico do solo $(0,92 ; \mathrm{p}<0,04)$, a massa de agregados $>2,0 \mathrm{~mm}$
$(0,90 ; p<0,02)$ e o índice de estabilidade de agregados $(0,93 ; \mathrm{p}<0,01)$. De maneira geral, a maior 
subestimação do C-FLO ocorreu nos sistemas de manejo com alto potencial de acúmulo de C. Esse efeito, portanto, pode comprometer a avaliação da magnitude da proteção física da MO, quando comparados diferentes sistemas de manejo num mesmo tipo de solo.

A redução do revolvimento do solo e a presença de gramíneas intensificam a agregação do solo (Silva \& Mielniczuk, 1997; Conceição et al., 2005, 2008), a qual está diretamente relacionada à proteção física da MO (Golchin et al., 1994b, 1997; Conceição, 2006). Portanto, se o solo apresenta elevada massa de agregados $>2 \mathrm{~mm}$ e alto IEA, com o uso de peneira de 9,5 mm, os agregados mantêm-se inteiros, e a MO oclusa em seu interior é exposta apenas após dispersão com ultrassom, para liberação da FLO. Ao contrário, quando se prepara uma amostra em peneira de $2 \mathrm{~mm}$, os agregados maiores que essa malha são quebrados pela moagem, e parte da FLO do interior dos agregados $>2 \mathrm{~mm}$ é exposta e quantificada como FLL. Dessa forma, quanto maior a quantidade de FLO protegida em agregados $>2 \mathrm{~mm}$ resistentes em água, maior será a superestimação da FLL, devido ao efeito do preparo inicial da amostra.

\section{Influência da subestimação do C-FLO na avaliação de sistemas de manejo do solo}

Nos cinco solos, as diferenças entre os dois sistemas de manejo avaliados diminuíram quando se utilizaram amostras $\leq 2,0 \mathrm{~mm}\left(2,18-2,97 \mathrm{~g} \mathrm{~kg}^{-1}\right.$ de $\mathrm{C}$ no solo), comparadas às amostras $\leq 9,5 \mathrm{~mm}\left(3,31-3,64 \mathrm{~g} \mathrm{~kg}^{-1}\right.$ de C no solo).

Ao comparar os sistemas de manejo com alto e baixo potencial de acúmulo de $\mathrm{C}$ em relação ao C-FLL e C-FLO, obtiveram-se resultados divergentes entre os dois preparos de amostra. Quando utilizada amostra $\leq 9,5 \mathrm{~mm}$, em todos os solos avaliados, os sistemas de manejo conservacionista proporcionaram aumento significativo na proteção física da MO, ou seja, acúmulo de C-FLO (Quadro 4). Entretanto, ao se utilizarem amostras $\leq 2,0 \mathrm{~mm}$, não foi possível verificar diferenças significativas no incremento de C-FLO em três dos solos avaliados (PV ES, LV-D e LV-M). Assim, a subestimação do C-FLO pelo uso de amostras $\leq 2,0 \mathrm{~mm}$ levaria à conclusão errônea de que a proteção física não seria um mecanismo importante de estabilização da MO no solo sob sistemas de manejo conservacionista (com alto potencial de acúmulo de C).

\section{CONCLUSÕES}

1. A utilização de amostras $\leq 2,0 \mathrm{~mm}$ no fracionamento físico da MOS subestima o teor de C protegido em agregados, sendo esse efeito mais pronunciado nos solos e sistemas de manejo com maior acúmulo de $\mathrm{C}$ e estabilidade dos agregados.

2. A subestimação da fração leve-oclusa pelo uso de amostras $\leq 2,0 \mathrm{~mm}$ pode, em alguns solos, levar à interpretação errônea de que a proteção física da MO não é um mecanismo eficiente na estabilização do $\mathrm{C}$ no solo.

3. Em estudos de proteção física da MO é recomendado o uso de amostras com tamanho inicial $\leq 9,5 \mathrm{~mm}$, ou, alternativamente, o uso da mesma peneira utilizada no preparo da amostra para avaliação da estabilidade dos agregados do solo, a qual normalmente é superior a $8 \mathrm{~mm}$.

\section{AGRADECIMENTOS}

Os autores agradecem à Embrapa Agropecuária Oeste e ao grupo SLC Agrícola, por disponibilizarem os experimentos sob sua responsabilidade para realização da pesquisa; À Capes, pela bolsa de PósDoutorado; e ao CNPq e à Fapergs, pelo apoio financeiro.

\section{LITERATURA CITADA}

BAYER, C.; MARTIN-NETO, L.; MIELNICZUK, J. \& PAVINATO, A. Armazenamento de carbono em frações lábeis da matéria orgânica de um Latossolo Vermelho sob plantio direto. Pesq. Agropec. Bras., 39:677-683, 2004.

BAYER, C.; MARTIN-NETO, L.; MIELNICZUK, J.; PAVINATO, A. \& DIECKOW, J. Carbon sequestration in two Brazilian Cerrado soils under no-till. Soil Tillage Res., 86:237-245, 2006.

BOENI, M. Proteção física da matéria orgânica em solos de Cerrado afetada pela pastagem. Porto Alegre, Universidade Federal do Rio Grande do Sul, 2007. 136p. (Tese de Doutorado)

CHRISTENSEN, B.T. Physical fractionation of soil and organic matter in primary particle size and density separates. Adv. Soil Sci., 20:1-90, 1992.

CONCEIÇÃO, P.C.; AMADO. T.J.C.; MIELNICZUK, J. \& SPAGNOLLO, E. Qualidade do solo em sistemas de manejo avaliada pela dinâmica da matéria orgânica e atributos relacionados. R. Bras. Ci. Solo, 29:777-788, 2005.

CONCEIÇAO, P.C. Agregação e proteção física da matéria orgânica em solos tropicais e subtropicais. Porto Alegre, Universidade Federal do Rio Grande do Sul, 2006. 138p. (Tese de Doutorado)

CONCEIÇÃO, P.C.; BOENI, M.; DIECKOW, J.; BAYER, C.; MARTIN-NETO, L. \& MIELNICZUK, J. Eficiência do politungstato de sódio no fracionamento físico densimétrico da matéria orgânica do solo. R. Bras. Ci. Solo, 31:1301-1310, 2007.

CONCEIÇÃO, P.C.; BOENI, M.; DIECKOW, J.; BAYER, C. \& MIELNICZUK, J. Fracionamento densimétrico com politungstato de sódio no estudo da proteção física da matéria orgânica do solo. R. Bras. Ci. Solo, 32:541-549, 2008. 
DIECKOW, J.; MIELNICZUK, J.; KNICKER, H.; BAYER, C.; DICK, D.P. \& KÖGEL-KNABNER, I. Carbon and nitrogen stocks en physical fractions of a subtropical acrisol as influenced by long-term no-till cropping systems and N fertilisation. Plant Soil, 268:319-328, 2005.

FREIXO, A.A.; MACHADO, P.L.O.A.; GUIMARÃES, C.M.; SILVA, C.A. \& FADIGAS, F.S. Estoques de carbono e nitrogênio e distribuição de frações orgânicas de Latossolo do Cerrado sob diferentes sistemas de cultivo. R. Bras. Ci. Solo., 26:425-434, 2002 .

GOLCHIN, A.; OADES, J.M.; SKJEMSTAD, J.O. \& CLARK, P. Study of free and occluded particulate organic matter in soils by solid state ${ }^{13} \mathrm{C}$ CP/MAS NMR spectroscopy and scanning electron microscope. Austr. J.. Soil Res., 32:285309, 1994a.

GOLCHIN, A.; OADES, J.M.; SKJEMSTAD, J.O. \& CLARKE, P. Soil-structure and carbon cycling. Austr. J. Soil Res., 32:1043-1068, 1994b.

GOLCHIN, A.; BALDOCK, J.A. \& OADES, J. A model linking organic matter decomposition, chemistry and aggregates dynamics. In: LAL, R. ed. Soil processes and the carbon cycle. Boca Raton, CRC Press, 1997. p.245-266.

INDA JUNIOR, A.V.; BAYER, C.; CONCEIÇÃO, P.C.; BOENI, M.; SALTON, J.C. \& TONIN, A.T. Variáveis relacionadas à estabilidade de complexos organo-minerais em solos tropicais e subtropicais brasileiros. Ci. Rural, 37:1301-1307, 2007.

LÜTZOW, M.; KÖGEL-KNABNER, I.; EKSCHMITT, K.; FLESSA, H.; GUGGENBERGER, G.; MATZNER, E. \& MARSCHNER, B. SOM fractionation methods: Relevance to functional pools and to stabilization mechanisms. Soil Biol. Biochem., 39:2183-2207, 2007.

MADARI, B.; MACHADO, P.L.O.A.; TORRES, E.; ANDRADE, A.G. \& VALENCIA, L.I.O. No tillage and crop rotation effects on soil aggregation and organic carbon in a Rhodic Ferralsol from southern Brazil. Soil Tillage Res., 80:185200, 2005 .
OADES, J.M. \& WATERS, A.G. Aggregate hierarchy in soils. Austr. J. Soil Res., 29:815-828, 1991.

ROSCOE, R. \& BUURMAN, P. Tillage effects on soil organic matter in density fractions of a cerrado Oxisol. Soil Tillage Res., 70:107-119, 2003.

ROSCOE, R. \& MACHADO, P.L.O.A. Fracionamento físico do solo em estudos da matéria orgânica. Dourados, Embrapa Agropecuária Oeste, 2002. 86p.

ROSCOE, R.; MADARY, B.E. \& MACHADO, P.L.O.A. Fracionamento físico do solo na obtenção de compartimentos mensuráveis para uso em simuladores da dinâmica da matéria orgânica. In: ROSCOE, R.; MERCANTE, F.M. \& SALTON, J.C. Dinâmica da matéria orgânica do solo em sistemas conservacionistas. Dourados, Embrapa, 2006. p.17-42

SALTON, J.C.; MIELNICZUK, J.; BAYER, C.; BOENI, M.; CONÇEIÇÃO, P.C.; FABRICIO, A.C.; MACEDO, M.C.M. \& BROCH, D.L. Agregação e estabilidade de agregados do solo em sistemas agropecuários em Mato Grosso do Sul. R. Bras. Ci. Solo, 32:11-21, 2008.

SILVA, I.F.; MIELNICZUK, J. Ação do sistema radicular de plantas na formação e estabilização de agregados do solo. R. Bras. Ci. Solo, 21:113-117, 1997.

SKJEMSTAD, J.O.; CLARKE, P.; TAYLOR, J.A.; OADES,J.M. $\&$ MCCLURE, S.G. The chemistry and nature of protected carbon in soil. Austr. J. Soil Res., 34:251-276, 1996.

TOMAZI, M. Estabilidade da matéria orgânica em Latossolos do Cerrado sob sistemas de uso e manejo. Porto Alegre, Universidade Federal do Rio Grande do Sul, 2008. 106p. (Tese de Doutorado) 
